\title{
Efeitos da implantação de técnicas agropecuárias na intensificação de sistemas de produção de leite em estabelecimentos familiares ${ }^{1}$
}

\section{Airton Manzano ${ }^{2}$, Nelson José Novaes ${ }^{2}$, Artur Chinelato de Camargo², Sérgio Novita Esteves ${ }^{2}$, Alfredo Ribeiro de Freitas ${ }^{2,3}$}

\author{
1 Projeto parcialmente financiado pela FAPESP. \\ ${ }^{2}$ Embrapa Pecuária Sudeste, C.P. 339, São Carlos, SP, CEP: 13.560 - 970. \\ ${ }^{3}$ Bolsista do CNPq.
}

RESUMO - A viabilidade econômica da produção de leite em estabelecimentos familiares (EF) foi avaliada no município de São Carlos, SP, no período de 1998 a 2001, por meio de ações técnicas e gerenciais que intensificam os sistemas de produção sustentável. Utilizaram-se seis EF (4,2 a 27,2 ha) que tinham como principal atividade econômica a produção de leite. As ações foram: agropecuárias: a) alimentação: plantio e fornecimento de cana-de-açúcar + uréia na seca, alimentação suplementar de acordo com a produção da vaca e pastejo rotacionado nas águas; b) manejo: melhoria do conforto dos animais; c) reprodução: uso de inseminação artificial e/ou monta natural e controle reprodutivo do rebanho; d) sanidade: controle de ecto e endoparasitas, exames de brucelose e tuberculose; e) melhoramento genético: uso de touro selecionado; ambientais: recuperação e conservação de recursos naturais, melhoria da qualidade da água e recuperação da fertilidade do solo; e gerenciais: controle zootécnico do rebanho, práticas de associativismo, descarte de animais improdutivos e análise econômica. Para a análise de variância em cada $\mathrm{EF}$, considerou-se um modelo que incluiu o efeito médio geral e os efeitos de ano e de meses dentro de ano. Uma vez que o efeito de ano esteve confundido com as ações técnicas e/ou tecnologias implementadas, as estimativas desse efeito, por polinômios ortogonais, possibilitaram estimar as mudanças quantitativas dos índices zootécnicos e gerenciais, em relação a 1998. As médias de acréscimos dos valores estimados dos seis EF em 2001, em comparação ao ano de 1998, para as produções de leite/ano, leite/ha/ano e leite/vaca, a receita e o patrimônio aumentaram $24,36,38,70$ e $27 \%$, respectivamente. Os custos operacional e total reduziram, respectivamente, 24,1 e $15,8 \%$ e o lucro aumentou $4 \%$. Entre os seis EF estudados, dois tornaram-se modelos de sistemas de produção de leite familiares. Concluiu-se que os pacotes tecnológicos implantados foram eficientes nos diferentes sistemas de produção de leite familiares.

Palavras-chave: ações tecnológicas, agricultura familiar, bovinos de leite, custo/benefício, lucro

\section{Effects of applying agricultural techniques for increase production of small family farms}

ABSTRACT - The economic viability of milk production in small family farms (SFF) in São Carlos, SP, from 1998 to 2001, after implementation of several technical and management actions in order to increase production was studied. Six SFF with sizes varying from 4.2 to 27.2 hectares and milk production as the main economical activity were selected. The technical actions implemented in each SFF were: agricultural: a) feeding: cropping and feeding sugar-cane + urea in the dry-season, concentrate supplementation according to the cow milk production level and rotational grazing in the rainy-season, b) animal welfare: identification and improvement of animal welfare, c) reproduction: use of artificial insemination and/or bulls and reproductive control of the herd, d) disease control: control of ectoparasites and endoparasites as well as brucellosis and tuberculosis tests, and e) genetic breeding: use of selected bulls, environment: recovery and conservation of natural resources, improvement of water quality and recovery of soil fertility, and administration techniques: culling of low-production animals, herd productivity control, practice of partnership and economical analysis. For statistical analysis was used an ANOVA model that included effects of the six SFF and fixed effects of year and months within year. The year effect was confounded with the technical and management actions and was assessed by orthogonal polynomials in order to estimate the quantitative changes in the response variables related to the year of 1998. The estimated means of the six SFF for annual milk production, milk production/ha/year, milk production/cow, net income and assets increased by $24 \%, 36 \%, 38 \%, 70 \%$ and $27 \%$, respectively comparing year 2001 with 1998. Operational and total costs reduced $24.1 \%$ and $15.8 \%$, respectively, while profit increased $4 \%$. Two out of six SFF were chosen as extension units and used as models of SFF. It was concluded that implementation of technologies were efficient in all SFF investigated in this study.

Key Words: dairy cattle, profit, cost/benefit, technical actions, small family farms 


\section{Introdução}

Segundo o Censo Agropecuário 1995/96 (Guanziroli \& Cardim, 2000), existem no Brasil 4,8 milhões de estabelecimentos rurais, ocupando área de 353,6 milhões de ha. O valor bruto da produção (VBP) agropecuária na safra deste período foi de $\mathrm{R} \$ 47,8$ bilhões e o financiamento total de $\mathrm{R} \$ 3,7$ bilhões. A agricultura familiar está presente em 4,1 milhões destes estabelecimentos $(85,2 \%)$, sendo responsável por $30,5 \%$ da área total e por $\mathrm{R} \$ 18,1$ bilhões, ou seja, $37,9 \%$ do VBP total. Contudo, esses estabelecimentos receberam apenas R $\$ 937$ milhões de financiamento rural, aproximadamente $25,3 \%$ do financiamento destinado à agricultura. A Região Sudeste, que possui 634 mil estabelecimentos familiares - EF (75,3\% do total), ocupando $29,2 \%$ da área regional e produzindo $24,4 \%$ de todo VBP da região, recebeu neste mesmo período apenas $12,6 \%$ do valor dos financiamentos agrícolas.

Com a criação do Programa Nacional de Fortalecimento da Agricultura Familiar - PRONAF, em 1995/96, visando aumentar a capacidade produtiva, a geração de emprego e renda e melhorar a qualidade de vida dos agricultores familiares, foram assinados, no período de 1995 a 1999, em torno de 2,4 milhões de contratos de financiamentos, resultando em empréstimo de mais seis bilhões de reais, o que melhorou significativamente os recursos financeiros aplicados neste segmento (Mattei, 2001).O orçamento da agricultura familliar para a safra 2004/2005 foi de R $\$ 7$ bilhões, enquanto para a safra de 2003/2004 foi de $\mathrm{R} \$ 5$,4 bilhões do plano. Esse recurso possibilitou ampliar de 1,4 milhão para 1,8 milhão o número de famílias de agricultores atendidas por políticas de crédito rural (Evangelista, 2004).

Em âmbito nacional, os agricultores de sistemas familiares produzem $52 \%$ do VBP total da pecuária de leite, $24 \%$ da pecuária de corte, $58 \%$ de suínos e $40 \%$ de aves e ovos, o que demonstra a importância deste segmento nos produtos destinados ao mercado interno e também entre os principais produtos de exportação agrícola brasileira. $\mathrm{Na}$ Região Sudeste, cuja média dos EF é de 30,0 ha, os agricultores são responsáveis por $22,5 \%$ da pecuária de corte, $37,5 \%$ da pecuária de leite, $21,0 \%$ de suínos e $17,8 \%$ de aves, estando a pecuária de leite presente em $44,1 \%$ das propriedades, a pecuária de corte em $27,9 \%$, a produção de suínos em $23,5 \%$ e a de aves e ovos em $53,4 \%$. Nessa região, a renda total dos EF é diversificada: $15 \%$ dos estabelecimentos apresentaram renda anual negativa ou nula; $55 \%$ entre zero e $\mathrm{R} \$ 3.000,00 ; 20 \%$ de $\mathrm{R} \$ 3.000,00$ a $\mathrm{R} \$ 8.000,00 ; 6 \%$ de $\mathrm{R} \$ 8.000,00 \mathrm{aR} \$ 15.000,00 ; 3 \% \mathrm{de} \mathrm{R} \$ 15.000,00 \mathrm{aR} \$ 27.500,00$; e 2\% mais de R $\$ 27.500,00$ (Guanziroli \& Cardim, 2000).
De acordo com Bortoleto et al. (1996), a Região Sudeste detém $47 \%$ da produção de leite do país. No estado de São Paulo, essa atividade está presente em 120 mil das 300 mil propriedades rurais, de modo que $80 \%$ dos produtores de leite ocupam de 3,1 a 100 ha e, destes, a metade não emprega qualquer forma de trabalho assalariado. Seguindo a tendência brasileira, estes sistemas têm baixa produtividade e participação decrescente na oferta global de leite; porém, pequena proporção tem produtividade média a alta, com participação crescente na oferta. Quanto à produção, em L/animal/dia, $80 \%$ dos sistemas produzem até 6,$0 ; 15 \%$, entre 6,1 e 8,0 ; e 5\% acima de 8,0 (Moricochi et al., 1994).

Com a abertura dos mercados e a conseqüente pressão concorrencial, a atividade leiteira precisa ser competitiva em custos e qualidade nos diferentes sistemas de produção do país e também frente à pecuária de outros países (Farina, 1996). Entretanto, vários fatores interferem no rendimento desta atividade: a) somente $16 \%$ do rebanho nacional é alimentado com rações balanceadas, sendo o consumo médio muito baixo (1 kg:16 litros); b) a inseminação artificial é usada em apenas $3 \%$ das vacas brasileiras em condições de reprodução; c) a mão-de-obra especializada é escassa; d) o nível tecnológico e organizacional de grande parte dos produtores é baixo, dificultando a ação da assistência técnica, reflexo da resistência à idéia de associação e de cooperação na gestão de seus negócios; e) falta de dados consistentes e realistas do setor.

$O$ fortalecimento da agricultura familiar requer que conceitos ultrapassados, como os da agricultura de baixa renda e da pequena produção ou da agricultura de subsistência, sejam eliminados, pois têm prejudicado o processo de integração desses agricultores no mercado competitivo.

A agricultura familiar deve ser entendida como o segmento que detém poder de influência econômica e social. Esta atividade, cujo capital pertence à família e em que a direção do processo produtivo está assegurada pelos proprietários, apesar do tamanho das unidades produtivas e de sua capacidade geradora de renda, possui características inteiramente compatíveis com a importante participação na oferta agrícola (Abramovay, 1997).

O dinamismo da agricultura familiar depende de sua base material de produção, sobretudo a fertilidade das terras, a formação dos agricultores e o ambiente sócio-econômico em que atuam, destacando-se o acesso diversificado aos mercados, ao crédito, à informação, à compra de insumos e aos meios materiais de exercícios de cidadania, como escola, saúde, assistência técnica, entre outros (Abromovay, 1997).

Verifica-se que tecnologias caras, embora excludentes aos agricultores familiares, dentro de certos limites de sua 
lógica, podem encontrar espaços, principalmente se apoiados nas diretrizes da sustentabilidade da produção agrícola (Carmo, 1998).

Este estudo foi realizado com o objetivo de viabilizar economicamente a produção de leite, por meio de ações técnicas e gerenciais de intensificação dos sistemas de produção, sob o enfoque sistêmico e de forma sustentável, em estabelecimentos familiares no município de São Carlos, SP.

\section{Material e Métodos}

O município de São Carlos está localizado na região central do estado de São Paulo, com população de aproximadamente 200 mil habitantes (mais de $90 \%$ na zona urbana e $10 \%$ na zonal rural) e taxas de crescimento anual de $2,74 \mathrm{e}$ $0,48 \%$, respectivamente. Produz em torno de $120.000 \mathrm{~L}$ de leite/dia e as propriedades rurais com áreas de 10 a 50 ha ocupam cerca de $46 \%$ da área rural e têm a pecuária leiteira como atividade principal, com produções de 20 a $150 \mathrm{~L} /$ dia.

Foram selecionados oito EF, cujos nomes e respectivas áreas, em ha, são: Sítio Planalto, 27,2(EF1); Chácara Taquari, 15 (EF2); Sítio Alvorada, 18(EF3); Chácara São Miguel, 4,2 (EF4); Estância São Judas Tadeu, 17 (EF5); Sítio São Bento, 14 (EF6); Sítio Jaraguá, 11,1 (P7); eEstância São Rafael, 11,9(P8). Após a seleção dos $\mathrm{EF}$, foi aplicado a cada produtor um questionário abrangendo os aspectos técnico, ambiental, social e econômico, para caracterizar o perfil tecnológico dos estabelecimentos. Posteriormente, foram definidas, de comum acordo com os produtores, as tecnologias a serem implantadas nos EF.

As técnicas escolhidas foram desenvolvidas por diferentes órgãos de pesquisa e reunidas em um pacote tecnológico (conjunto ordenado de técnicas) envolvendo as seguintes ações: agropecuária: a) alimentação: plantio e fornecimento de cana-de-açúcar + uréia na época da seca, alimentação suplementar de acordo com a produção da vaca e pastejo rotacionado na época das águas com o uso de cerca elétrica; b) manejo: identificação e melhorias no conforto dos animais (sombra), na distribuição de água e na qualidade do leite (higiene na ordenha, instalações e resfriamento do leite); c) reprodução: uso de inseminação artificial e/ou monta natural e controle reprodutivo do rebanho; d) sanidade: controle de ectoparasitas e endoparasitas e exames de brucelose e tuberculose; e) melhoramento genético: uso de touro selecionado; ambiental: recuperação e conservação de recursos naturais, melhoria da qualidade da água e recuperação da fertilidade do solo; e gerencial: controle leiteiro, descarte de animais improdutivos, controle zootécnico do rebanho, práticas de associativismo, análise econômica por meio de planilha de custo constituída de: receitas, despesas de custeio e investimentos, resultados zootécnicos e econômicos com depreciações de máquinas, instalações e remuneração de capital, animais e terra, elaborada pelos pesquisadores envolvidos no projeto.

$\mathrm{Na}$ determinação do custo operacional, foram consideradas as despesas relativas ao custeio na produção do leite e, na determinação do custo total, os custos fixos, como depreciação de máquinas, equipamentos e instalações (vida útil de dez anos), e a remuneração do capital investido em animais e na terra ( $6 \%$ ao ano).

O acompanhamento do projeto foi realizado pelos pesquisadores no período de 1998 a 2001, por meio de visitas quinzenais aos EF. Os dados foram coletados nas propriedades pelos proprietários e/ou pesquisadores utilizando-se gabaritos específicos. A eficiência dos tratamentos (incorporação de tecnologias agropecuárias disponíveis) foi medida em cada $E F$ por meio das mudanças qualiquantitativas dos índices zootécnicos, econômicos e gerenciais no período em relação ao diagnóstico inicial realizado em 1998.

A primeira análise consistiu da elaboração de tabelas de contingência utilizando-se dados obtidos do questionário aplicado em 1998, de modo a caracterizar o perfil tecnológico dos estabelecimentos em estudo. Na segunda análise, as variáveis produção de leite, (L/ano e L/ha/ano), custo operacional ( $R \$ / L)$, custo total $(R \$ / L)$, receita ( $R$ \$ano), despesas (R $\$ / a n o)$, lucro (R $\$ / a n o$ e $R$ \$/ha/ano), variação patrimonial (R\$), produção (L/vaca/ano) e total de animais/ ano, avaliadas mensalmente durante os anos de 1998 a 2001, foram avaliadas por análises de variância e regressão. Inicialmente, adotou-se o seguinte modelo de efeito fixo de ano: $y_{i j k}=\mu+A_{i}+\varepsilon_{j(i)}$, em que $y_{i j k}=o$ valor observado no k-ésimo $E F$, no j-ésimo mês do i-ésimo ano; $\mu=o$ efeito médio geral; $\mathrm{A}_{\mathrm{i}}=$ efeito do ano; e $\varepsilon_{\mathrm{j}(\mathrm{i})=}$ erro aleatório do modelo, com $\mathrm{i}=1,2,3,4 ; \mathrm{j}=1,2, \ldots, 12 \mathrm{e} \mathrm{k}=1,2, \ldots, 6$. Os três graus de liberdade associados ao efeito de ano foram desdobrados em linear, quadrático e cúbico por meio de polinômios ortogonais, de modo a estimar o efeito das ações técnicas e/ou tecnologias implementadas nos índices zootécnicos e gerenciais, via análise de regressão. As análises foram implementadas com o auxílio do pacote computacional SAS (SAS, 2000).

\section{Resultados e Discussão}

\section{Diagnóstico inicial dos estabelecimentos familiares}

Todos os produtores eram alfabetizados e dois possuíam curso superior (Tabela 1); a mão-de-obra (familiar e não-familiar) variava de 3 a 9 pessoas. Este excesso de 
mão-de-obra possivelmente se deve ao manejo inadequado dos animais, confirmado pela baixa produção de leite obtida, que será discutida posteriormente. Os EF7 e EF8 foram eliminados por não terem atendido à metodologia do trabalho. Assim, na discussão que se segue, a denominação EF1 a EF6 será usada na identificação de produtor, estabelecimento familiar e propriedade.

\section{Características gerais das propriedades}

Todas possuíam estábulos e picadeiras e o solo era preparado mecanicamente; cinco tinham tratores, quatro possuíam botijões de sêmen e três dispunham de resfriadores de leite (Tabela 2). As práticas conservacionistas eram realizadas em quatro propriedades; três realizavam análise de solo e somente duas utilizavam sementes e mudas selecionadas.

\section{Características zootécnicas das propriedades}

Em todas as propriedades, utilizavam-se ordenha mecânica e cana-de-açúcar como alimentação suplementar e efetuavam-se vacinação contra aftosa e brucelose e controle de parasitas. Em todas, praticava-se inseminação artificial e o rebanho era constituído de animais cruzados Holandês $\mathrm{x}$ Zebu, dividido em vacas em lactação e secas, novilhas e bezerras. Entretanto, não se utilizavam irrigação e pastejo rotacionado e a escrituração era realizada de modo incipiente.

Tabela 1 - Escolaridade do produtor e mão-de-obra utilizada no estabelecimento familiar Table 1 - Farmer educational level and type of labor used in the small family farm

\begin{tabular}{|c|c|c|c|c|c|c|}
\hline & \multicolumn{6}{|c|}{$\begin{array}{c}\text { Estabelecimento } \\
\text { Farm }\end{array}$} \\
\hline & EF 1 & EF2 & EF 3 & EF4 & EF 5 & EF 6 \\
\hline Reside na propriedade (Lives in the farm) & $\mathrm{S}$ & $\mathrm{S}$ & $\mathrm{S}$ & $\mathrm{S}$ & $\mathrm{S}$ & $\mathrm{S}$ \\
\hline Estudou até o 10 grau (Finished elementary school) & - & $\mathrm{S}$ & - & - & - & $\mathrm{S}$ \\
\hline Estudou até o 2o grau (Finished high school) & $\mathrm{S}$ & - & - & $\mathrm{S}$ & - & - \\
\hline Superior (Children with undergraduate degree) & - & - & $\mathrm{S}$ & - & - & $\mathrm{S}$ \\
\hline Filhos com nível superior (Undergraduate degree) & - & $\mathrm{S}$ & - & - & - & $\mathrm{S}$ \\
\hline Mão-de-obra familiar (Family labor) & - & - & - & - & - & - \\
\hline Homem/Ano (Man/Year) & 1 & 1 & 1 & 2 & 4 & 1 \\
\hline Mulher/Ano (Woman/Year) & - & 1 & - & 2 & 1 & 1 \\
\hline Mão-de-obra extra familiar (Hired labor) & - & - & - & - & - & - \\
\hline Permanente, homem/ano (Full time, man/year) & 3 & 3 & 2 & - & 4 & 1 \\
\hline Temporária, dias/ano (Temporary, days/year) & - & - & 100 & - & - & - \\
\hline
\end{tabular}

$\mathrm{S}=\operatorname{sim}($ yes); "-” informação não disponível (information not available).

Tabela 2 - Áreas, benfeitorias, máquinas e equipamentos dos estabelecimentos familiares

Table 2 - Sizes, buildings, barns, fences, machines and equipments of the small family farms

\begin{tabular}{|c|c|c|c|c|c|c|}
\hline & \multicolumn{6}{|c|}{$\begin{array}{c}\text { Estabelecimento } \\
\text { Farm }\end{array}$} \\
\hline & EF 1 & EF2 & EF 3 & EF 4 & EF 5 & EF 6 \\
\hline Área, ha $($ Size, ha) & 27,2 & 5,0 & 15,3 & 3,5 & 17 & 14,0 \\
\hline Área arrendada, ha (Rented area, ha) & & 10,0 & & 0,7 & & \\
\hline Conservação de solo (Soil conservation) & $\mathrm{N}$ & S & $\mathrm{S}$ & $\mathrm{S}$ & $\mathrm{S}$ & $\mathrm{N}$ \\
\hline Análise de solo (Soil analysis) & $\mathrm{N}$ & $\mathrm{S}$ & $\mathrm{N}$ & $\mathrm{S}$ & $\mathrm{S}$ & $\mathrm{N}$ \\
\hline Utiliza sementes/mudas melhoradas (Uses improved seeds and moulting) & $\mathrm{N}$ & $\mathrm{N}$ & $\mathrm{S}$ & $\mathrm{S}$ & $\mathrm{N}$ & $\mathrm{S}$ \\
\hline \multicolumn{7}{|l|}{ Preparo do solo (Soil management) } \\
\hline Tração animal (Animal) & $\mathrm{N}$ & $\mathrm{N}$ & $\mathrm{N}$ & $\mathrm{S}$ & $\mathrm{N}$ & $\mathrm{S}$ \\
\hline Tração mecânica (Mechanical) & $\mathrm{S}$ & $\mathrm{S}$ & $\mathrm{S}$ & $\mathrm{S}$ & $\mathrm{S}$ & $\mathrm{S}$ \\
\hline \multicolumn{7}{|l|}{ Benfeitorias e construção (Buildings and barns) } \\
\hline Estábulo (Barn) & $\mathrm{S}$ & $\mathrm{S}$ & $\mathrm{S}$ & $\mathrm{S}$ & $\mathrm{S}$ & $\mathrm{S}$ \\
\hline Silo (Silo) & $\mathrm{S}$ & $\mathrm{S}$ & $\mathrm{N}$ & $\mathrm{S}$ & $\mathrm{N}$ & $\mathrm{N}$ \\
\hline Curral (Yard) & $\mathrm{S}$ & $\mathrm{S}$ & $\mathrm{N}$ & $\mathrm{N}$ & $\mathrm{N}$ & $\mathrm{N}$ \\
\hline \multicolumn{7}{|l|}{ Máquinas e equipamentos (Machines and equipments) } \\
\hline Aparelho de cerca elétrica (Electrical fence equipment) & $\mathrm{S}$ & $\mathrm{S}$ & $\mathrm{S}$ & $\mathrm{N}$ & $\mathrm{N}$ & $\mathrm{N}$ \\
\hline Balança para ração (Scale for feed) & $\mathrm{S}$ & $\mathrm{S}$ & $\mathrm{S}$ & $\mathrm{N}$ & $\mathrm{S}$ & $\mathrm{N}$ \\
\hline Botijão de sêmen (Bottle of semen) & $\mathrm{S}$ & $\mathrm{S}$ & $\mathrm{S}$ & $\mathrm{N}$ & $\mathrm{S}$ & $\mathrm{N}$ \\
\hline Resfriador de leite (Milk cooler) & $\mathrm{S}$ & $\mathrm{S}$ & $\mathrm{N}$ & $\mathrm{N}$ & $\mathrm{S}$ & $\mathrm{N}$ \\
\hline Trator (Tractor) & $\mathrm{S}$ & $\mathrm{S}$ & $\mathrm{S}$ & $\mathrm{N}$ & $\mathrm{S}$ & $\mathrm{S}$ \\
\hline Picadeira (Grass cutting machine) & $\mathrm{S}$ & $\mathrm{S}$ & $\mathrm{S}$ & $\mathrm{S}$ & $\mathrm{S}$ & $\mathrm{S}$ \\
\hline
\end{tabular}

$\mathrm{S}=\operatorname{sim}($ yes $), \mathrm{N}=$ não $($ no). 
A produção média diária de leite era de 33 a 784 litros e a produção por área, de 3.428 a $16.833 \mathrm{~L} / \mathrm{ha} / \mathrm{ano}$, indicando que os produtores tinham potencial para aumentar as produções (Tabela 3).

Caracteristicas gerais dos produtores e das propriedades

A metade dos produtores entregava o leite na cooperativa e a outra, onde pagava-se melhor pelo leite. $\mathrm{O}$ baixo preço constituía-se no principal problema (Tabela 4).

Uma análise global do diagnóstico inicial dos EF permite afirmar que, embora os produtores estivessem realizando práticas como inseminação artificial, escrituração zootécnica, alimentação dos animais com cana-de-açúcar, entre outras, não obtiveram os resultados esperados, possivelmente por falta de orientação técnica e de políticas de financiamento. Além disso, desconheciam os procedimentos para a escolha adequada do sêmen e das melhores variedades de cana-deaçúcar. Quanto à escrituração zootécnica, faziam somente anotações de coberturas e nascimentos. Apenas uma cooperativa prestava assistência técnica e, quanto aos financiamentos para custeio e investimento, três produtores utilizaram este tipo de serviço.

Consta na Tabela 5 um resumo dos resultados das análises de regressão, com estimativas dos coeficientes linear, quadrático e cúbico do modelo polinomial ortogonal ajustado para cada EF, respectivas significâncias e coeficientes de determinação $\left(\mathrm{R}^{2}\right)$ dos modelos. Uma vez que o efeito de ano esteve confundido com as ações técnicas e/ou tecnologias implementadas e, considerando-se que todas as equações foram significativas $(\mathrm{P}<0,05)$, as estimativas obtidas por meio dessas equações são alternativas para se obter resposta aproximada das mudanças qualiquantitativas dos índices zootécnicos e gerenciais em relação ao diagnóstico inicial de 1998. Uma equação polinomial ortogonal ajustada satisfatoriamente representa uma função resposta aproximada válida.

Tabela 3 - Características zootécnicas dos estabelecimentos familiares

Table 3 - Productivity characteristics of the small family farms

\begin{tabular}{|c|c|c|c|c|c|c|}
\hline & \multicolumn{6}{|c|}{$\begin{array}{c}\text { Estabelecimento } \\
\text { Farm }\end{array}$} \\
\hline & EF 1 & EF2 & EF 3 & EF4 & EF 5 & EF 6 \\
\hline Inseminação artificial (Artificial insemination) & $\mathrm{S}$ & $\mathrm{S}$ & $\mathrm{S}$ & $\mathrm{S}$ & $\mathrm{S}$ & $\mathrm{N}$ \\
\hline Idade ao $1^{\circ}$ parto, mês (Age at first calving, month) & 33 & 33 & 27 & 36 & 24 & 36 \\
\hline \multicolumn{7}{|l|}{ Tipo de bezerreiro (Calf barn) } \\
\hline Individual (Single) & $\mathrm{S}$ & $\mathrm{S}$ & $\mathrm{N}$ & $\mathrm{N}$ & $\mathrm{S}$ & $\mathrm{S}$ \\
\hline Coletivo (Collective) & $\mathrm{S}$ & $\mathrm{N}$ & $\mathrm{S}$ & $\mathrm{S}$ & $\mathrm{N}$ & $\mathrm{N}$ \\
\hline \multicolumn{7}{|l|}{ Local de ordenha (Milking location) } \\
\hline Estábulo (Barn) & $\mathrm{N}$ & $\mathrm{S}$ & $\mathrm{N}$ & $\mathrm{S}$ & $\mathrm{N}$ & $\mathrm{S}$ \\
\hline Sala de ordenha (Milking parlour) & $\mathrm{S}$ & $\mathrm{N}$ & $\mathrm{S}$ & $\mathrm{N}$ & $\mathrm{S}$ & $\mathrm{N}$ \\
\hline Tipo de ordenha (Milking procedure) & $\mathrm{N}$ & $\mathrm{N}$ & $\mathrm{S}$ & $\mathrm{N}$ & $\mathrm{N}$ & $\mathrm{N}$ \\
\hline Mecânica (Mechanical) & $\mathrm{S}$ & $\mathrm{S}$ & $\mathrm{S}$ & $\mathrm{S}$ & $\mathrm{S}$ & $\mathrm{S}$ \\
\hline Manual (Manual) & $\mathrm{N}$ & $\mathrm{N}$ & $\mathrm{N}$ & $\mathrm{N}$ & $\mathrm{N}$ & $\mathrm{N}$ \\
\hline Divisão do rebanho em lotes (Division of herd in lots) & $\mathrm{S}$ & $\mathrm{S}$ & $\mathrm{S}$ & $\mathrm{S}$ & $\mathrm{S}$ & $\mathrm{N}$ \\
\hline \multicolumn{7}{|l|}{ Alimentação suplementar (Use of supplements) } \\
\hline Capineira (Grass paddock) & $\mathrm{S}$ & $\mathrm{N}$ & $\mathrm{N}$ & $\mathrm{S}$ & $\mathrm{N}$ & $\mathrm{N}$ \\
\hline Cana-de-açúcar (Sugar cane) & $\mathrm{S}$ & $\mathrm{S}$ & $\mathrm{S}$ & $\mathrm{S}$ & $\mathrm{S}$ & $\mathrm{S}$ \\
\hline Silagem (Silage) & $\mathrm{N}$ & $\mathrm{S}$ & $\mathrm{N}$ & $\mathrm{S}$ & $\mathrm{N}$ & $\mathrm{S}$ \\
\hline Suplemento mineral (Mineral supplement) & $\mathrm{S}$ & $\mathrm{S}$ & $\mathrm{S}$ & $\mathrm{S}$ & $\mathrm{S}$ & $\mathrm{S}$ \\
\hline \multicolumn{7}{|l|}{ Vacinação dos animais (Animal vaccination) } \\
\hline Aftosa (Aftosa) & $\mathrm{S}$ & $\mathrm{S}$ & $\mathrm{S}$ & $\mathrm{S}$ & $\mathrm{S}$ & $\mathrm{S}$ \\
\hline Brucelose (Brucellosis) & $\mathrm{S}$ & $\mathrm{S}$ & $\mathrm{S}$ & $\mathrm{S}$ & $\mathrm{S}$ & $\mathrm{S}$ \\
\hline Carbúnculo (Clostridium) & $\mathrm{S}$ & $\mathrm{S}$ & $\mathrm{N}$ & $\mathrm{S}$ & $\mathrm{N}$ & $\mathrm{N}$ \\
\hline Exames de tuberculose e brucelose (Brucellosis and tuberculosis tests) & $\mathrm{S}$ & $\mathrm{S}$ & $\mathrm{N}$ & $\mathrm{N}$ & $\mathrm{N}$ & $\mathrm{N}$ \\
\hline Controle de ecto e endoparasitas (Ecto and endo-parasites control) & $\mathrm{S}$ & $\mathrm{S}$ & $\mathrm{S}$ & $\mathrm{S}$ & $\mathrm{S}$ & $\mathrm{S}$ \\
\hline Escrituração zootécnica (Productivity bookkeeping) & $\mathrm{N}$ & $\mathrm{N}$ & $\mathrm{N}$ & $\mathrm{N}$ & $\mathrm{N}$ & $\mathrm{N}$ \\
\hline Uso de pastejo rotacionado (Use of rotational grazing) & $\mathrm{N}$ & $\mathrm{N}$ & $\mathrm{N}$ & $\mathrm{N}$ & $\mathrm{N}$ & $\mathrm{N}$ \\
\hline Uso de irrigação (Use of irrigation) & $\mathrm{N}$ & $\mathrm{N}$ & $\mathrm{N}$ & $\mathrm{N}$ & $\mathrm{N}$ & $\mathrm{N}$ \\
\hline Total de vacas (Total of cows) & 50 & 39 & 30 & 9 & 54 & 43 \\
\hline Vacas em lactação (Lactating cows) & 40 & 27 & 23 & 4 & 44 & 30 \\
\hline Novilhas (Heifers) & 50 & 25 & 20 & 6 & 26 & 1 \\
\hline Identificação dos animais (Identification of the animals) & $\mathrm{S}$ & $\mathrm{N}$ & $\mathrm{N}$ & $\mathrm{N}$ & $\mathrm{N}$ & $\mathrm{N}$ \\
\hline Produção diária de leite, L (Daily milk production, L) & 427 & 498 & 274 & 33 & 784 & 260 \\
\hline Produção de leite, L/ha/ano (Milk production, L/ha/year) & 6118 & 12133 & 6536 & 3428 & 16833 & 6785 \\
\hline Produção de leite, L/vaca total/dia (Milk production, L/total cow/day) & 8,5 & 12,7 & 9,1 & 3,7 & 14,5 & 6,0 \\
\hline Produção de leite, L/vaca/dia (Milk production, L/ cow/day) & 10,7 & 18,4 & 11,9 & 8,2 & 17,8 & 8,7 \\
\hline$\%$ de vacas em lactação (\% of lactating cows) & 80,0 & 69,2 & 76,7 & 44,4 & 81,5 & 69,7 \\
\hline
\end{tabular}

$\mathrm{S}=\operatorname{sim}($ yes), $\mathrm{N}=$ não $($ no $)$. 
Tabela 4 - Características gerais dos produtores e dos estabelecimentos familiares

Table 4 - General characteristics of the farmers and the small family farms

\begin{tabular}{|c|c|c|c|c|c|c|}
\hline & \multicolumn{6}{|c|}{$\begin{array}{c}\text { Estabelecimento } \\
\text { Farm }\end{array}$} \\
\hline & EF 1 & EF 2 & EF3 & EF4 & EF 5 & EF6 \\
\hline \multicolumn{7}{|l|}{ Entrega do leite (Milk deliver) } \\
\hline Cooperativa (Cooperative) & $\mathrm{S}$ & $\mathrm{N}$ & $\mathrm{S}$ & $\mathrm{N}$ & $\mathrm{N}$ & $\mathrm{S}$ \\
\hline Outros (Others) & $\mathrm{N}$ & $\mathrm{S}$ & $\mathrm{N}$ & $\mathrm{S}$ & $\mathrm{S}$ & $\mathrm{S}$ \\
\hline \multicolumn{7}{|l|}{ Por que? (Why?) } \\
\hline Melhor preço (Better price) & $\mathrm{N}$ & $\mathrm{S}$ & $\mathrm{N}$ & $\mathrm{S}$ & $\mathrm{S}$ & $\mathrm{N}$ \\
\hline Não tem outra escolha (No other choice) & $\mathrm{S}$ & $\mathrm{N}$ & $\mathrm{S}$ & $\mathrm{N}$ & $\mathrm{N}$ & $\mathrm{S}$ \\
\hline Outros (Others) & $\mathrm{N}$ & $\mathrm{N}$ & $\mathrm{S}$ & $\mathrm{S}$ & $\mathrm{N}$ & $\mathrm{N}$ \\
\hline \multicolumn{7}{|l|}{ Principais problemas enfrentados (Main problems) } \\
\hline Preço baixo do produto (Low milk price) & $\mathrm{S}$ & $\mathrm{S}$ & $\mathrm{S}$ & $\mathrm{S}$ & $\mathrm{N}$ & $\mathrm{S}$ \\
\hline Preço alto dos insumos (High price of inputs) & $\mathrm{N}$ & $\mathrm{S}$ & $\mathrm{N}$ & $\mathrm{S}$ & $\mathrm{S}$ & $\mathrm{S}$ \\
\hline Preço alto da mão-de-obra (High price of labor) & $\mathrm{S}$ & $\mathrm{N}$ & $\mathrm{N}$ & $\mathrm{N}$ & $\mathrm{N}$ & $\mathrm{S}$ \\
\hline Falta de mão-de-obra especializada (Lack of specialized labor) & $\mathrm{S}$ & $\mathrm{N}$ & $\mathrm{N}$ & $\mathrm{N}$ & $\mathrm{N}$ & $\mathrm{S}$ \\
\hline Falta de informação (Lack of information) & $\mathrm{S}$ & $\mathrm{N}$ & $\mathrm{N}$ & $\mathrm{S}$ & $\mathrm{N}$ & $\mathrm{N}$ \\
\hline Falta de financiamento (Lack of financing) & $\mathrm{S}$ & $\mathrm{S}$ & $\mathrm{N}$ & $\mathrm{S}$ & $\mathrm{N}$ & $\mathrm{S}$ \\
\hline \multicolumn{7}{|l|}{ Instituições que procura para apoio técnico } \\
\hline \multicolumn{7}{|l|}{ Institutions used for technical help } \\
\hline Secretaria de Agricultura Municipal (Country Agricultural Secretary) & $\mathrm{N}$ & $\mathrm{N}$ & $\mathrm{N}$ & $\mathrm{N}$ & $\mathrm{N}$ & $\mathrm{N}$ \\
\hline Cooperativas (Cooperatives) & $\mathrm{S}$ & $\mathrm{S}$ & $\mathrm{S}$ & $\mathrm{N}$ & $\mathrm{N}$ & $\mathrm{N}$ \\
\hline CATI (State extension service) & $\mathrm{N}$ & $\mathrm{N}$ & $\mathrm{N}$ & $\mathrm{N}$ & $\mathrm{S}$ & $\mathrm{N}$ \\
\hline Bancos (Banks) & $\mathrm{N}$ & $\mathrm{N}$ & $\mathrm{N}$ & $\mathrm{N}$ & $\mathrm{N}$ & $\mathrm{S}$ \\
\hline Outras (Others) & $\mathrm{N}$ & $\mathrm{N}$ & $\mathrm{N}$ & $\mathrm{S}$ & $\mathrm{N}$ & $\mathrm{N}$ \\
\hline \multicolumn{7}{|l|}{ Assistência técnica nos últimos 12 meses } \\
\hline \multicolumn{7}{|l|}{ Technical assistance in the last twelve months } \\
\hline Mensal (Monthly) & $\mathrm{N}$ & $\mathrm{S}$ & $\mathrm{S}$ & $\mathrm{N}$ & $\mathrm{S}$ & $\mathrm{N}$ \\
\hline Acima de um mês (Less than once a month) & $\mathrm{N}$ & $\mathrm{N}$ & $\mathrm{N}$ & $\mathrm{S}$ & $\mathrm{N}$ & $\mathrm{N}$ \\
\hline Não recebeu (No technical assistance) & $\mathrm{S}$ & $\mathrm{N}$ & $\mathrm{N}$ & $\mathrm{N}$ & $\mathrm{N}$ & $\mathrm{S}$ \\
\hline Conhece algum tipo de financiamento? (Do you know any kind of financing?) & $\mathrm{N}$ & $\mathrm{S}$ & $\mathrm{S}$ & $\mathrm{N}$ & $\mathrm{S}$ & $\mathrm{S}$ \\
\hline Acesso a algum tipo de financiamento (Access to financing) & $\mathrm{N}$ & $\mathrm{S}$ & $\mathrm{S}$ & $\mathrm{N}$ & $\mathrm{S}$ & $\mathrm{S}$ \\
\hline \multicolumn{7}{|l|}{ Finalidade do financiamento } \\
\hline \multicolumn{7}{|l|}{ Objective of financing } \\
\hline Custeio (Expense) & $\mathrm{N}$ & $\mathrm{S}$ & $\mathrm{S}$ & $\mathrm{N}$ & $\mathrm{N}$ & $\mathrm{S}$ \\
\hline Investimento (Investment) & $\mathrm{N}$ & $\mathrm{N}$ & $\mathrm{S}$ & $\mathrm{N}$ & $\mathrm{S}$ & $\mathrm{S}$ \\
\hline \multicolumn{7}{|l|}{ Entidade de solicitação de financiamento } \\
\hline \multicolumn{7}{|l|}{ Where finance was requested } \\
\hline Banco (Bank) & $\mathrm{N}$ & $\mathrm{S}$ & $\mathrm{N}$ & $\mathrm{N}$ & $\mathrm{S}$ & $\mathrm{N}$ \\
\hline Outras (Others) & $\mathrm{N}$ & $\mathrm{N}$ & $\mathrm{S}$ & $\mathrm{N}$ & $\mathrm{N}$ & $\mathrm{N}$ \\
\hline \multicolumn{7}{|l|}{ Quitou o financiamento (Paid loan) } \\
\hline $\operatorname{Sim}($ Yes $)$ & $\mathrm{N}$ & $\mathrm{S}$ & $\mathrm{S}$ & $\mathrm{N}$ & $\mathrm{N}$ & $\mathrm{N}$ \\
\hline Não, devido aos altos encargos (No, due to high charges) & $\mathrm{N}$ & $\mathrm{N}$ & $\mathrm{N}$ & $\mathrm{N}$ & $\mathrm{N}$ & $\mathrm{S}$ \\
\hline Ainda está pagando (Still paying) & $\mathrm{N}$ & $\mathrm{N}$ & $\mathrm{N}$ & $\mathrm{N}$ & $\mathrm{S}$ & $\mathrm{N}$ \\
\hline
\end{tabular}

$\mathrm{S}=\operatorname{sim}($ yes $), \mathrm{N}=$ não $($ no) .

Embora as técnicas propostas tenham produzido resultados satisfatórios já no primeiro ano de sua implantação, a discussão dos resultados foi feita considerandose os resultados alcançados em três anos (1999 a 2001). Este prazo, no entanto, foi insuficiente para que todo o pacote tecnológico implantado apresentasse os resultados esperados. Projetos desta natureza necessitam de pelo menos cinco anos para promover os resultados desejados, pois parte deste tempo é utilizada pelo produtor no aprendizado de atividades como preenchimento das planilhas econômicas e zootécnicas, no preparo, na correção e adubação do solo, na recuperação de pastagens e no plantio de forrageiras como os capins mombaça, tanzânia, napier, coastcross, tifton e cana-de-açúcar, atividades que requerem um tempo entre o plantio e seu uso na alimentação dos animais.

Quanto à produção de leite/ano, em litros (Figura 1A), houve acréscimos significativos $(\mathrm{P}<0,05)$, estimados por análise de regressão, em cinco propriedades, à exceção do estabelecimento 5 (EF5), que, em razão da venda de parte das vacas em lactação, obteve redução na produção. Os acréscimos em 2001, em relação a 1998, obtidos com base nas estimativas, foram de $114,6,82,563 \mathrm{e} 48 \%$, respectivamente, para EF1, EF2, EF3, EF4 e EF6; para o EF5, houve redução de $64 \%$.

Da mesma forma, à exceção do EF5, as tecnologias implantadas nos EF proporcionaram acréscimos $(\mathrm{P}<0,05)$ nas produções de leite por área, L/ha/ano (Figura 1B), nas 
Tabela 5 - Estimativas de parâmetros de regressão polinomial e coeficiente de determinação $\left(R^{2}\right)$ associados aos seis estabelecimentos familiares (EF1 a EF6) de São Carlos, SP. O modelo de regressão ajustado é do tipo $Y=a+b x+c x^{2}+d x^{3}$, em que $Y$ é a variável dependente e $\mathrm{x}$, a variável independente (anos de 1998 a 2001)

Table 5 - Estimates of parameters and determination coefficients $\left(R^{2}\right)$ of orthogonal polynomial regression model adjusted to six small family farms (EF1 a EF2) in São Carlos, SP. The regression model is of type $Y=a+b x+c x^{2}+d x^{3}$, where $Y$ is the dependent variable and $X$ is associated to the years (1998 to 2001)

\begin{tabular}{|c|c|c|c|c|c|c|c|}
\hline \multirow[t]{2}{*}{$\begin{array}{l}\text { Característica }(\mathrm{Y}) \\
\text { Trait }\end{array}$} & & \multicolumn{6}{|c|}{$\begin{array}{c}\text { Estabelecimento } \\
\text { Farm }\end{array}$} \\
\hline & & EF 1 & EF2 & EF3 & EF 4 & EF 5 & EF 6 \\
\hline Produção de leite total/ano & a & $1.090,56$ & $14.990,00$ & $21.820,00$ & $-5.457,66$ & $53.984,00$ & $8.975,83$ \\
\hline \multirow[t]{4}{*}{ Total milk production/year } & $\mathrm{b}$ & $13.628,00$ & 328,47 & $-25.121,00$ & $9.632,24$ & $-36.593,00$ & $-1.790,30$ \\
\hline & $\mathrm{c}$ & $-1.738,06$ & & $13.558,00$ & $-3.622,16$ & $15.101,00$ & 611,08 \\
\hline & $\mathrm{d}$ & & & $-1.923,24$ & 497,59 & $-2.074,70$ & \\
\hline & $\mathrm{R}^{2}$ & 0,95 & 0,78 & 1,00 & 1,00 & 1,00 & 0,89 \\
\hline Produção de leite/ha/ano & a & 484,69 & $11.992,00$ & $14.546,00$ & - & $38.106,00$ & $7.693,57$ \\
\hline \multirow[t]{6}{*}{ Milk production/ha/year } & $\mathrm{b}$ & $6.057,10$ & 262,77 & $-16.747,00$ & $15.593,00$ & - & $-1.534,54$ \\
\hline & c & $-772,47$ & & $9.038,97$ & $27.521,00$ & $25.830,00$ & 523,78 \\
\hline & $\mathrm{d}$ & & & $-1.282,16$ & - & $10.659,00$ & \\
\hline & $\mathrm{R}^{2}$ & 0,83 & 0,82 & 1,00 & $10.349,00$ & $-1.464,50$ & 0,84 \\
\hline & & & & & $1.421,69$ & 1,00 & \\
\hline & & & & & 1,00 & & \\
\hline Custo operacional do leite, $\mathrm{R} \$$ & a & 0,52 & 0,22 & 0,43 & 2,04 & 0,16 & 0,12 \\
\hline \multirow[t]{4}{*}{ Loperational cost of milk, $R \$ / L$} & $\mathrm{~b}$ & $-0,17$ & 0,07 & $-0,11$ & $-2,18$ & 0,05 & 0,14 \\
\hline & c & 0,03 & & 0,02 & 0,82 & & $-0,03$ \\
\hline & $\mathrm{d}$ & & & & $-0,10$ & & \\
\hline & $\mathrm{R}^{2}$ & 0,77 & 0,72 & 0,76 & 1,00 & 0,71 & 0,78 \\
\hline Custo do leite, $\mathrm{R} \$ / \mathrm{L}$ & a & 0,98 & 0,36 & 0,11 & 2,80 & 0,18 & 0,17 \\
\hline \multirow[t]{4}{*}{ Milk cost, $R \$ / L$} & $\mathrm{~b}$ & $-0,72$ & $-0,02$ & 0,62 & $-2,90$ & 0,07 & 0,18 \\
\hline & $\mathrm{c}$ & 0,27 & 0,02 & $-0,31$ & 1,07 & & $-0,04$ \\
\hline & $\mathrm{d}$ & $-0,03$ & & 0,04 & $-0,12$ & & \\
\hline & $\mathrm{R}^{2}$ & 1,00 & 0,92 & 1,00 & 1,00 & 0,82 & 0,88 \\
\hline Receita,R\$ & $\mathrm{a}$ & $-3.297,26$ & $-534,42$ & $14.997,00$ & $-483,20$ & $23.357,00$ & $5.831,43$ \\
\hline \multirow[t]{4}{*}{ Income, $R \$$} & $\mathrm{~b}$ & $6.583,50$ & $9.163,49$ & $-18.386,00$ & 838,28 & $-21.696,00$ & $-5.235,09$ \\
\hline & $\mathrm{c}$ & $-763,91$ & $-1.531,24$ & $8.238,48$ & & $-1.337,72$ & $2.200,99$ \\
\hline & $d$ & & & $-1.058,27$ & & & $-255,34$ \\
\hline & $\mathrm{R}^{2}$ & 0,80 & 0,81 & 1,00 & 0,78 & 0,82 & 1,00 \\
\hline Despesas, R\$ & $\mathrm{a}$ & $8.828,57$ & $3.327,64$ & $14.997,00$ & $1.272,83$ & $20.409,00$ & $6.431,92$ \\
\hline \multirow[t]{4}{*}{ Expenses, $R \$$} & $\mathrm{~b}$ & $-3.876,41$ & $4.066,89$ & $-18.386,00$ & $-620,18$ & $-20.443,00$ & $-6.330,93$ \\
\hline & $\mathrm{c}$ & $1.399,50$ & $-2.420,22$ & $8.238,48$ & 203,85 & $9.252,14$ & $2.784,75$ \\
\hline & $\mathrm{d}$ & & 470,69 & $-1.058,27$ & & $-1.248,94$ & $-366,75$ \\
\hline & $\mathrm{R}^{2}$ & 0,83 & 1,00 & 1,00 & 0,84 & 1,00 & 1,00 \\
\hline Lucro, R\$ & $\mathrm{a}$ & $-1.212,00$ & $-75,89$ & $-9.689,97$ & $-1.364,02$ & 2034,73 & 569,34 \\
\hline \multirow[t]{4}{*}{ Profit, $R \$$} & $\mathrm{~b}$ & $1.046,00$ & $-950,75$ & $10.756,00$ & $1.054,39$ & 205,56 & $-764,75$ \\
\hline & $\mathrm{c}$ & $-2.163,42$ & $3.623,43$ & $3.450,53$ & $-122,43$ & $-197,67$ & 251,83 \\
\hline & d & & $-838,79$ & 342,43 & & & \\
\hline & $\mathrm{R}^{2}$ & 0,88 & 1,00 & 1,00 & 0,86 & 0,78 & 0,82 \\
\hline
\end{tabular}



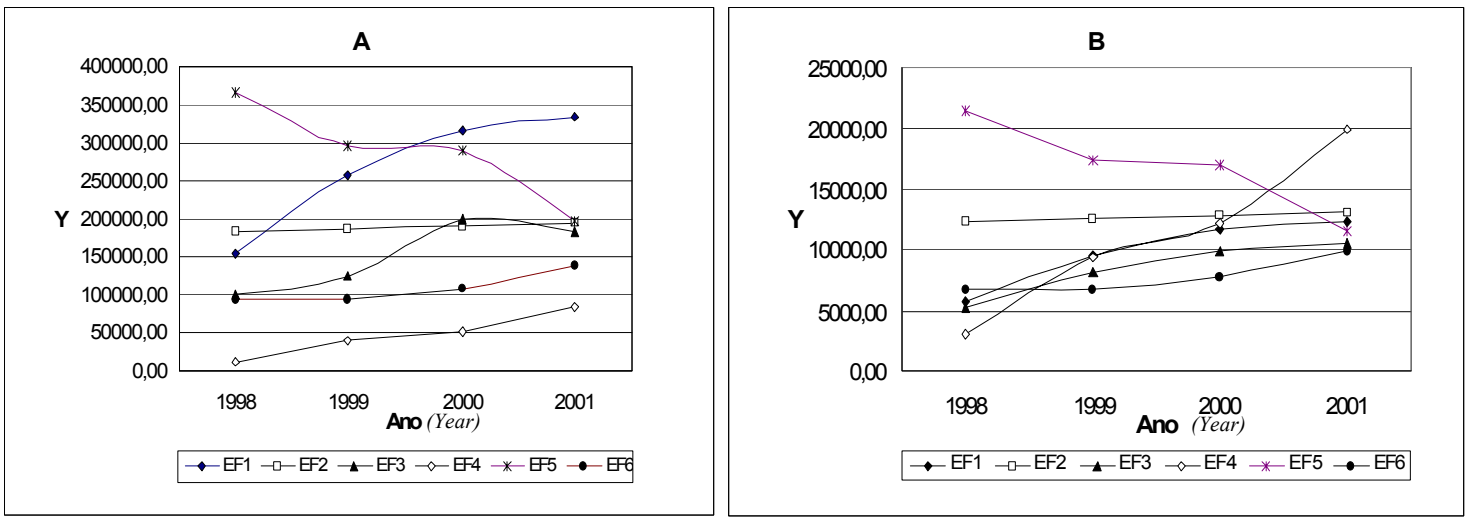

Figura 1 - Produção de leite/ano (A) e de leite/ha/ano (B) em seis estabelecimentos familiares (EF1 a EF6) do município de São Carlos, SP.

Figure 1 - Milk production/year (A) and milk production/ha/year (B) of six small family farms (EF1 to EF6) in São Carlos, SP.
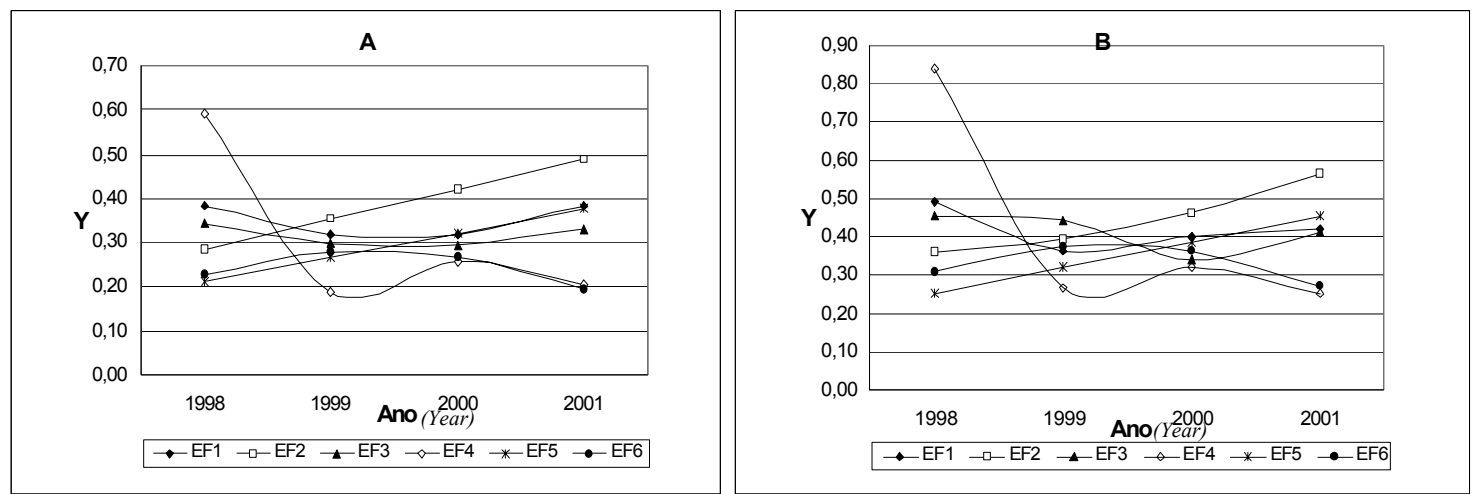

Figura 2 - Média do custo operacional do leite, em $R \$ / L(A)$, e média do custo total do leite, $R \$ / L$ (B) em seis estabelecimentos familiares (EF1 a EF6) do município de São Carlos, SP.

Figure 2 - Average of operational cost of milk, in $R \$ / L(A)$ and average of total costs of milk, in $R \$ / L(B)$ of six small family farms (EF1 to EF6) in São Carlos, SP.

proporções observadas para produção de leite anual (Figura 1A). Entretanto, a média dos valores estimados da produção em 2001 nos seis estabelecimentos aumentou $(\mathrm{P}<0,05)$ em 131\% em relação ao ano de 1998.

As variações ocorridas na produção de leite/ano e na produção/ha/ano nos diferentes $\mathrm{EF}$ são decorrentes de fatores como: condição cultural e disponibilidade de recursos financeiros do produtor, qualidade genética do rebanho, área da propriedade e velocidade de implantação das técnicas propostas. Entretanto, constatou-se que o pacote tecnológico proposto foi eficiente, independentemente dos sistemas de produção utilizados.

$\mathrm{Na}$ determinação do custo operacional (Figura 2A), foram consideradas somente as despesas relativas aos insumos necessários à produção do leite. Houve aumento $(\mathrm{P}<0,05)$ no custo operacional nos EF2 e EF5, reflexo da maior aplicação de insumos, principalmente de alimentos concentrados. Em 2001, a participação dos insumos na despesa foi de 44, 41, 35, 49, 39 e 21\%, nos sistemas EF1 a
EF6, respectivamente. Com exceção do EF5, no qual houve redução de $20 \%$ no número de vacas, nos demais $\mathrm{EF}$, a ingestão de concentrado proporcionou aumento da produção de leite (Figura 1A).

Com base nos valores estimados pela regressão, verificou-se redução $(\mathrm{P}<0,05)$ no custo operacional para $E F 3$, EF4 e EF6 e acréscimos $(\mathrm{P}<0,05)$ para EF2 e EF5 (Figura 2A).

No EF4, particularmente, houve decréscimo no custo operacional e aumento na produção de leite, evidenciando que esse produtor, no início do trabalho, necessitava de conhecimentos em práticas de gestão e manejo de animais, hipótese confirmada pelos resultados obtidos no primeiro ano (1999) de desenvolvimento do projeto (Figura 1A). Os resultados obtidos por este produtor após três anos indicam que ele assimilou e implantou rapidamente o pacote tecnológico proposto. Em média, o custo operacional diminuiu em 13\%.

A introdução de ações gerenciais para controle de custos foi importante, pois proporcionou aos produtores condições de avaliação dos custos de produção do leite. 

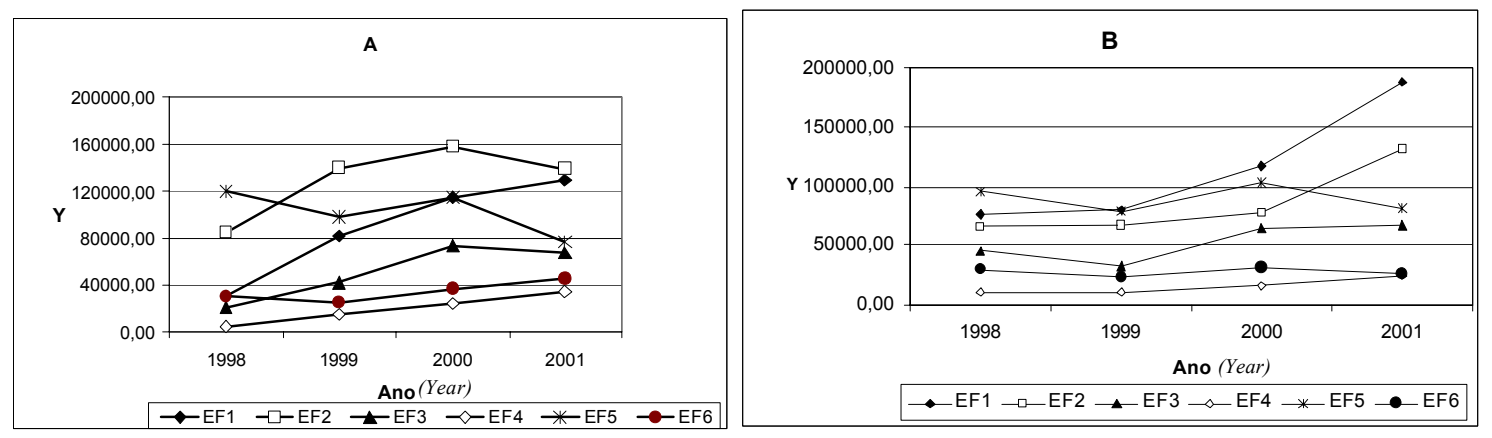

Figura 3 - Receitas (A) e despesas (B), em $R \$ / L$, de seis estabelecimentos familiares (EF1 a EF6) do município de São Carlos, SP. Figure 3 - Income $(A)$ and expenses (B), in $R \$ / L$, of six small family farms (EF1 to EF6) in São Carlos, $S P$.

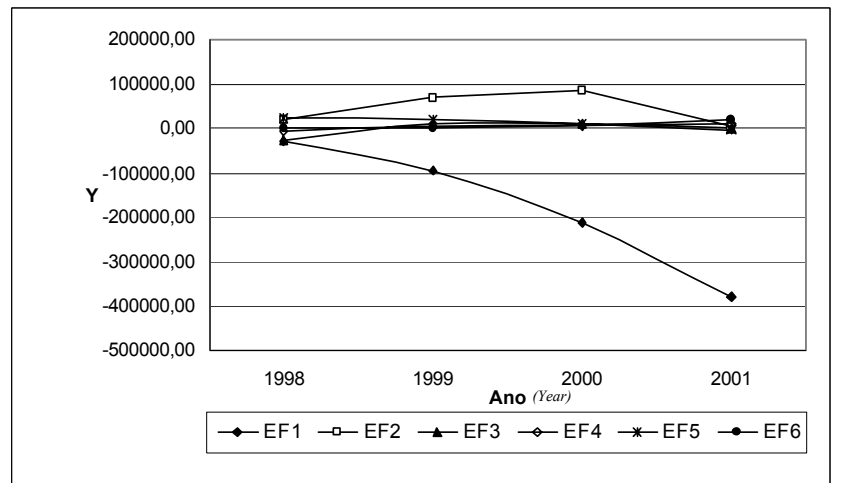

Figura 4 - Lucro, em $\mathrm{R} \$$, de seis estabelecimentos familiares de São Carlos, SP.

Figure 4 - Profit in $R \$$ of six small family farms in São Carlos, SP.

O conhecimento da remuneração do capital total investido na atividade leiteira (custo fixo) e de sua influência no custo final do leite alertou os produtores quanto à importância dos investimentos serem realizados com racionalidade, uma vez que podem alcançar níveis elevados e influenciar negativamente a atividade leiteira.

Quanto aos custos totais, considerando a remuneração do capital e as depreciações (Figura 2B), os resultados obtidos apresentaram as mesmas tendências daquelas observadas com o custo operacional. Verifica-se que, nos $\mathrm{EF} 1, \mathrm{EF} 3$, EF4 e EF6, houve redução $(\mathrm{P}<0,05)$ nos custos após três anos de implantação das técnicas propostas, enquanto, nos EF2 e EF5, houve aumento $(\mathrm{P}<0,05)$. À exceção de EF5, os aumentos de custos foram compensados com os de produção de leite; em média, os custos totais diminuíram 5\% nas propriedades.

No início do projeto, a média do custo fixo nos estabelecimentos representava $24,4 \%$ do custo total. Com a execução do projeto, houve aumento do custo operacional e, conseqüentemente, do custo total. Entretanto, como o custo fixo permaneceu constante, sua participação em relação ao custo total no final do projeto foi reduzida para $16,0 \%$.
Após três anos de implantação das técnicas propostas (Figura 3A), com exceção de EF5, houve aumentos significativos $(\mathrm{P}<0,05)$ nas receitas e, na média dos valores observados em 2001, o aumento $(\mathrm{P}<0,05)$ foi de $222 \%$ em relação a 1998. As receitas estão relacionadas aos aumentos na produção de leite e na quantidade de animais comercializados, decorrentes das ações gerencias e agropecuárias adotadas pelos produtores. Outro aspecto a ser ressaltado é que, no período de três anos, o preço/litro de leite recebido pelos produtores aumentou em média $38 \%$.

Quando comparado ao ano de 1998 (aplicação do questionário), houve aumentos $(\mathrm{P}<0,05)$ de despesas nos estabelecimentos familiares EF1, EF2, EF3 e EF4 (Figura 3B). Entretanto, a média de aumento $(\mathrm{P}<0,05)$ nas seis propriedades foi de $68 \%$. O aumento de receita, como esperado, foi acompanhado de aumentos de despesas.

A participação percentual dos principais insumos nas despesas em 2001 foi de $37,7 \%$ de concentrado $(21 \%=E F 6$ a $49 \%=\mathrm{EF} 4) ; 8,4 \%$ de fertilizantes $(6 \%=\mathrm{EF} 3$ a $14 \%=\mathrm{EF} 4) ; 5 \%$ de energia elétrica $(4 \%=\mathrm{EF} 5$ a $10 \%=\mathrm{EF} 6) ; 4,9 \%$ de combustível $(4 \%=\mathrm{EF} 1$ a $7 \%=\mathrm{EF} 6) ; 4 \%$ de medicamentos $(2 \%=\mathrm{EF} 2$ a $7 \%=\mathrm{EF} 1) ; 3,2 \%$ desal mineral $(1 \%=\mathrm{EF} 6 \mathrm{a} 4 \%=\mathrm{EF} 1) ; \mathrm{e} 1,8 \%$ de manutenção de equipamentos $(1 \%=\mathrm{EF} 6$ a $4 \%=\mathrm{EF} 1)$. No caso da mão-de-obra, a média foi de $15 \%$ quando considerada apenas aquela permanente e não-familiar (zero $=\mathrm{EF} 4$ a $28 \%=$ EF6). Esses itens corresponderam a $80 \%$ das despesas, confirmando os dados obtidos por Rodrigues Filho et al. (2002).

As receitas estão relacionadas aos aumentos de produção de leite e da quantidade de animais comercializados, decorrentes das ações gerencias e agropecuárias adotadas pelos produtores. Outro aspecto a ser ressaltado é que, no período de três anos, o preço/litro de leite recebido pelos produtores aumentou, em média, $38 \%$.

Quanto ao lucro (receita - despesa), nos estabelecimentos familiares EF1, EF3 e EF4, a produção de leite gerava prejuízo em 1998 (Figura 4). Com a implantação do pacote tecnológico, 
passou a ser lucrativa $(\mathrm{P}<0,05)$ em EF3 e EF4 já no primeiro ano do projeto, possibilitando a realização de investimentos de R\$ 16.400,00 e R\$ 10.978,00, respectivamente, em animais, instalações, máquinas e equipamentos. No EF6, embora tenha apresentado lucro $(\mathrm{P}<0,05)$, não foram realizados investimentos; no EF2, houve lucro $(\mathrm{P}<0,05)$, mas as tecnologias implantadas mostraram seus efeitos positivos em 1999 e 2000, com decréscimo em 2001, explicado pelo investimento de R\$16.800,00, realizado em animais. Em EF1, o aumento na produção de leite permitiu investimentos de $\mathrm{R} \$ 14.965,00 \mathrm{em}$ 2000 e de R \$63.030,00 em 2001 (R \$53.500,00 para aquisição de animais), o que afetou significativamente $(\mathrm{P}<0,05)$ o lucro. A média de lucro dos EF comprovou aumento de 4\%.

O lucro/ha/ano e o aumento patrimonial observados nas planilhas de custo das diferentes propriedades (Tabela 6) confirmam os alcançados com o lucro/ano, ilustrados na Figura 4. Ressalta-se que o lucro/ha/ano, que estava negativo em EF4 (R\$ -1.645,70) em 1998, foi positivo em 2001 ( R \$2.930,00) em EF6, passando de $\mathrm{R} \$ 19,70$ para $\mathrm{R} \$ 1.348,00$ no mesmo período $(\mathrm{P}<0,05)$, ou seja, após a implantação do pacote tecnológico.
Após três anos de adoção das técnicas propostas, todos os produtores obtiveram melhorias $(\mathrm{P}<0,05)$ no patrimônio, destacando-se o EF2 e EF6, com aumentos de 56 e $41 \%$, respectivamente. Os menores aumentos foram observados para EF1 e EF5 (16 e 17\%), enquanto, para EF3 e EF4, os aumentos foram de 27 e $25 \%$, respectivamente. A média de aumento nos seis EF foi de $30 \%$.

$\mathrm{Na}$ determinação das médias de produção por vaca/ano (Tabela 7), considerou-se o total de vacas do rebanho, o que revela a eficiência do sistema de produção de leite, pois reflete os manejos alimentar, reprodutivo e sanitário do rebanho e está relacionado à rentabilidade da atividade leiteira.

A produção/vaca/ano aumentou $(\mathrm{P}<0,05)$ em todos os EF, exceto para EF5, pelos motivos já descritos. Houve aumento de $79 \%$ na média dos EF no período de 1998 a 001 , indicando que as ações técnicas e gerenciais atuaram positivamente nos sistemas de produção.

Houve aumento de $16 \%$ no número médio de vacas do rebanho entre 1998 e 2001 (Tabela 7), representado principalmente pelo EF1, em que houve aumento de 52\%. Entretanto, verificou-se também aumento de produção/vaca, compro-

Tabela 6 - Lucro/ha/ano e aumento patrimonial dos seis estabelecimentos familiares avaliados em São Carlos, SP Table 6 - Profit/ha/year and patrimony increase in six small family farms in São Carlos, SP

\begin{tabular}{|c|c|c|c|c|c|c|c|}
\hline & \multirow[t]{2}{*}{$\begin{array}{l}\text { Ano } \\
\text { Year }\end{array}$} & \multicolumn{6}{|c|}{$\begin{array}{c}\text { Estabelecimento } \\
\text { Farm }\end{array}$} \\
\hline & & EF 1 & EF2 & EF3 & EF 4 & EF 5 & EF 6 \\
\hline Lucro/ha/ano, R\$ & 1998 & $-1.672,40$ & $1.406,40$ & $-1.361,30$ & $-1.645,70$ & $1.459,70$ & 19,70 \\
\hline \multirow[t]{3}{*}{ Profit/ha/year, $R \$$} & 1999 & $-26,10$ & $4.644,80$ & 506,40 & $1.345,00$ & $1.114,60$ & 126,40 \\
\hline & 2000 & $-7,90$ & $5.628,20$ & 512,70 & $1.524,10$ & 669,50 & 378,25 \\
\hline & 2001 & $-2.207,70$ & 330,50 & 27,60 & $2.693,00$ & $-251,60$ & $1.348,30$ \\
\hline Patrimônio, R\$ & 1998 & $237.100,00$ & $110.240,00$ & $147.750,00$ & $44.900,00$ & $200.100,00$ & $97.300,00$ \\
\hline \multirow[t]{3}{*}{ Patrimony, $R \$$} & 1999 & $225.400,00$ & $112.120,00$ & $158.250,00$ & $43.150,00$ & $211.420,00$ & $112.280,00$ \\
\hline & 2000 & $269.100,00$ & $122.040,00$ & $150.150,00$ & $46.950,00$ & $207.420,00$ & $131.380,00$ \\
\hline & 2001 & $274.914,00$ & $172.140,00$ & $188.150,00$ & $56.026,00$ & $234.420,00$ & $137.000,00$ \\
\hline
\end{tabular}

Tabela 7 - Média de produção de leite, L/vaca, número de vaca/ano e total de animais/ano dos seis estabelecimentos familiares avaliados em São Carlos, SP

Table 7 - $\quad$ Average milk production, L/cow, number of cows/year and total number of animals/year of small family farms in São Carlos, SP

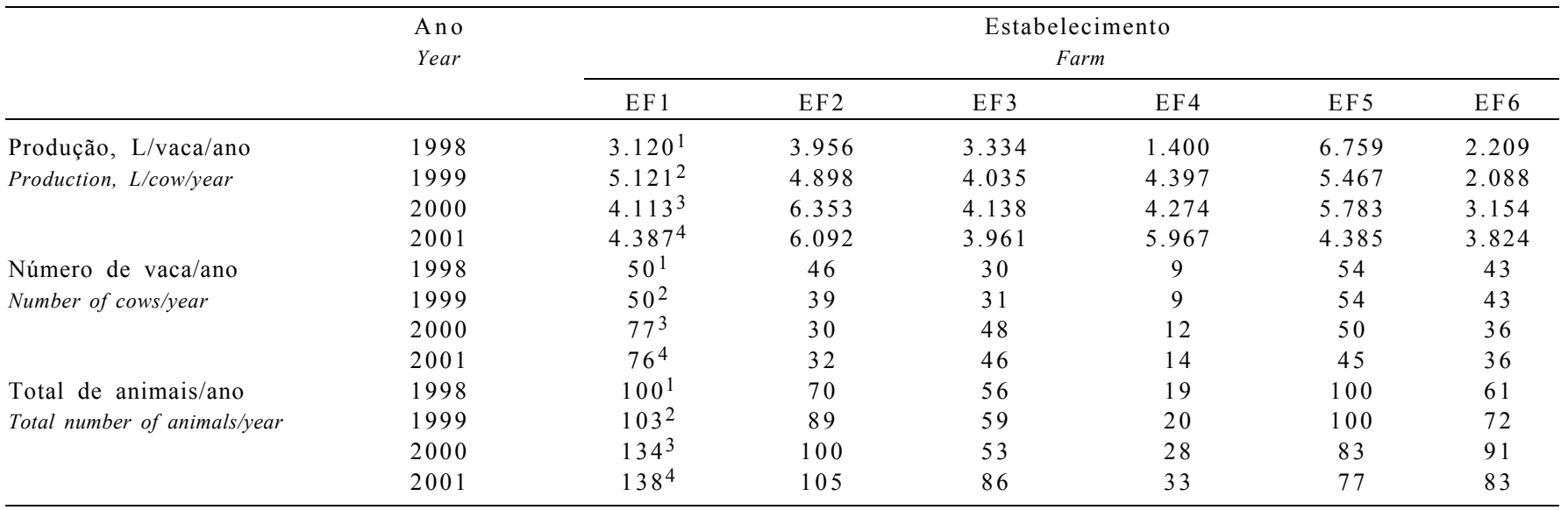


vando que as técnicas implantadas proporcionaram os efeitos desejados. Outro aspecto importante foi a média de aumento de $38 \%$ no total de animais nos rebanhos, indicando que o pacote tecnológico implantado, sobretudo o uso intensivo de pastagens (pastejo rotacionado) no período das águas e de cana-de-açúcar como alimento volumoso no período da seca, proporcionou aumento na capacidade de suporte das pastagens das propriedades.

Os dados obtidos neste trabalho corroboram a afirmativa de Rodrigues Filho et al. 2002 de que "mercado, pagamento diferenciado, escala de produção e estratégias de manejo alimentar constituem parâmetros indispensáveis ao estudo de viabilidade econômica para adoção de sistemas de produção".

\section{Conclusões}

Em média, houve aumentos de 128, 131, 79, 16, 38, 222 e $68 \%$, respectivamente, para as produções de leite/ano, de leite/ha/ano e de leite/vaca/ano, o número médio de vacas do rebanho, o total de animais, a receita e a despesa nos seis estabelecimentos familiares estudados. Verificaram-se, no entanto, acréscimos de apenas 12 e 5\% nos custos operacional e total.

Os custos operacionais foram afetados pelos seguintes insumos, em ordem decrescente de importância: alimentos concentrados, mão-de-obra extrafamiliar, fertilizantes, energia elétrica, combustível, medicamentos, sal mineral e manutenção de máquinas, que representaram $80 \%$ das despesas.

Dos seis EF estudados, dois tornaram-se unidades demonstrativas, servindo de modelos de sistemas familiares de produção de leite para a Região Sudeste.

O conjunto de técnicas implantadas nos diferentes sistemas de produção proporcionou melhorias significativas na produção de leite/ano, na produção de leite/ha/ano, na produção/vaca/ano, na receita e no aumento patrimonial dos estabelecimentos familiares.

\section{Literatura Citada}

ABromovay, R. Agricultura familiar e uso do solo. São Paulo em Perspectiva, v.11, n.2, p.5-10, 1997.

Bortoleto, E.E.; CROCETTA, I.; RAMOS, J. et al. Cadeia produtiva do leite no Estado de São Paulo. Repensado a Agricultura Paulista. São Paulo: Secretaria de Agricultura e Abastecimento do Estado de São Paulo, 1996. 61p.

BROWN JR., B.W. The crossover experiment for clinical trials. Biometric, v.36, n.1, p.69-79, 1980 .

CARMO, M.S. A produção familiar como lucus ideal da agricultura sustentável. Agricultura em São Paulo, v.45, n.1, p.1$15,1998$.

FARINA, E.M.M.Q. Mudanças na exploração e no mercado do leite. Balde Branco, v.33, n.75, p.34-39, 1996.

EVANGELISTA, G. 2004. Governo aumentará recursos para a agricultura familiar, informa secretário. Disponível em: www.radiobras.gov.br. Acesso em: 15/06/2004.

GUANZIROLI, C.E.; CARDIM, S.E.C.S. Novo retrato da agricultura familiar - O Brasil redescoberto. Projeto de Cooperação Técnica INCRA/FAO, 2000. 74p.

MATEI, L. Programa Nacional de Fortalecimento da Agricultura Familiar (PRONAF): concepção, abrangência e limites observados. In: ENCONTRO DA SOCIEDADE BRASILEIRA DE SISTEMAS DE PRODUÇÃO, 4., 2001, Belém. Anais... Belém: Sociedade Brasileira de Sistemas de Produção, 2001.

MORICOCHI, L.; FERREIRA, C.R.R.P.T.; FAGUNDES, L. Produção de leite no Estado de São Paulo: potencial tecnológico. Agricultura em São Paulo, v.41, n.2, p.141-157, 1994.

RODRIGUES FILHO, M.; MANCIO, A.B.; GOMES, A.T. et al. Avaliação econômica do confinamento de novilhos de origem leiteira, alimentados com diferentes níveis de concentrado e de cama de frango. Revista Brasileira de Zootecnia, v.31, n.5, p.2055-2069, 2002 .

STATISTICAL ANALYSES SYSTEM - SAS. SAS/INSIGHT User's Guide. versão 8.2, versão para Windows Cary: 2000. 